\title{
COMPARING VARIOUS CHANNEL ESTIMATION TECHNIQUES FOR OFDM SYSTEMS USING MATLAB
}

\begin{abstract}
This paper compares the performance of various channel estimation techniques for OFDM systems over quasi-static channels using MATLab. It compares the performance of five channel estimation techniques, these are: decision directed (DD), linear interpolation, second-order interpolation, discrete Fourier transform (DFT) interpolation, minimum mean square error (MMSE) interpolation. The performance is evaluated in terms of two widely-used performance measures, namely, bit-error rate (BER) and the mean square error (MSE) for different levels of signal-to-noise ratio (SNR). The OFDM model is explained and implemented using MATLab to run different simulations. The simulation results demonstrate that the DD channel estimation provides the lowest BER and MSE as compared to interpolation techniques, at the cost of extra processing delay and comparatively sensitive to channel variations between OFDM symbols. Also, the MMSE interpolation outperforms all other interpolation techniques.
\end{abstract}

Raghad K. Mohammed

Department of Basic Sciences, College of Dentistry

University of Baghdad, Baghdad, Iraq

\section{KEYWORDS}

OFDM, pilot-based channel estimation, pilot allocation, direct decision, interpolation channel estimation, LS, MMSE, MATLab

\section{INTRODUCTION}

Orthogonal frequency division multiplexing (OFDM) is a special case of multicarrier transmission and frequency division multiplexing, where a single data stream is transmitted over several lower rate subcarriers, placed orthogonal to each other [1-3]. In OFDM, the input stream is multiplexed into $N$ symbol streams, each with symbol period $T_{\mathrm{s}}$. Each symbol stream is used to modulate parallel, synchronous subcarriers. OFDM becomes one of the most favorable modulation techniques for modern wireless communication systems, as it can provide high data rates with optimum BER and sufficient robustness to radio channel impairments. This is due to its resistance to inter-symbol interference (ISI) and low-complexity, especially in high-speed data communication systems compared to traditional single technique [4].

OFDM has been adopted by many data communication standards, such as wireless LAN protocols (e.g., IEEE 802.11a, IEEE 802.11g, IEEE 802.11n, IEEE 802.11ac, and IEEE 802.11ad), WiMAX, long term evolution (LTE), LTE advanced 4G mobile phone standards, digital audio broadcasting (DAB), digital video broadcasting (DVB), digital television DVB-T/T2 (terrestrial), and modern narrow and broadband power line communications, etc. [5-8].

Channel estimation has been recognized as one of the main challenges to the wider use of OFDM systems. Therefore, an intensive research has been carried-out to develop accurate, efficient, reliable, and cost-effective channels estimation solutions for OFDM systems. In order to be able to trustfully select between these techniques, it is crucial to evaluate and compare the performance of the techniques under different systems and wireless communication 
environments. Performing these investigations in real environments is time consuming and costly. Thus, computer simulation is appeared as the only feasible solution to rely on to evaluate and compare the performance of these techniques [2, 3, 9-12].

There are three channel estimation concepts, these are: pilot-based (also known as trainingbased), blind, and semi-blind channel estimation concepts. In pilot-based channel estimation, pilot tones that are known a priori to the receiver are multiplexed along with the data stream for channel estimation. The blind channel estimation is carried-out by evaluating the statistical information of the channel and certain properties of the transmitted signals. Blind channel estimation has its advantage in that it has no overhead loss; however, it is only applicable to slowly time-varying channels due to its need for a long data record. Semi-blind channel technique is hybrid of blind and pilot technique, utilizing pilots and other natural constraints to perform channel estimation [13].

In this paper, we concern with pilot-based channel estimation techniques for OFDM systems over quasi-static channels. Five pilot-based channel estimation techniques are briefly described; these are: decision directed (DD), linear interpolation, second-order interpolation, discrete Fourier transform (DFT) interpolation, and minimum mean square error (MMSE) interpolation. A research-level MATLab simulation model is developed to evaluate and compare the performance of the above techniques. The performance of the different channel estimation techniques is evaluated in terms of two widely-used parameters, namely, the bit error rate (BER) and the mean square error (MSE) for various input signal-to-noise ratio (SNR) [14-16].

This paper is organized as follows: This section introduces the main theme of this paper. Section 2 reviews some of the most recent and related work on simulation and performance evaluation of OFDM systems using MATLab. Section 3 presents the basic principles of OFDM, and pilot allocation techniques. The channel estimation techniques over quasi-static channels for OFDM systems are presented in Section 4. The description of the OFDM model is explained in Section 5. Simulation results are presented and discussed in Section 6. Finally, in Section 7, conclusions and a number of recommendations for future work are presented.

\section{LITERATURE REVIEW}

This section presents some of the most recent work on the simulation and performance evaluation of OFDM systems using MATLab. A review on OFDM modulation technique and its applications are presented by Khosla et. al in [17]. Sinha et. al [14] developed and analyzed the performance of a MIMO orthogonal space time block coded OFDM system to ensure high data rate, simultaneously increase in capacity range, and maintain adequate wireless communication reliability.

Salehi et. al [18] introduced a new intelligent and different method for channel estimation using learning automata, entitled LA estimator, where the learning automata are search agents, and each pair is responsible for searching one coefficient of the channel frequency response. The method can attain accurate channel estimation with a moderate computational complexity in comparison with genetic algorithms (GA) and particle swarm optimization (PSO) estimators. Furthermore, with higher convergence rate, the method is capable of providing the same performance as GA and PSO.

Manhas and Soni [15] analyzed the performance of OFDM system using various fading channels and channel coding. They developed a MATLab simulation model to calculate the BER for 
different fading channels (e.g., AWGN, Rayleigh and Rician) for different digital modulation (e.g., BPSK, QPSK and QAM). Kaur and Gupta [19] explored two multiple access techniques, namely, OFDM and SC-FDMA (single carries frequency division multiple access) and implemented them in MATLab. They conclude that SC-FDMA with STBC is more effective and performs better than OFDM-STBC as it has lower BER as well as lesser PAPR (peak-to-average power ratio), which is a severe disadvantage of OFDM system. SC-FDMA-STBC reduces the particular limitations that came across the technique of OFDM-STBC.

Jain and Nandal [20] analyzed and compared the performance of channel estimation of MIMO communication systems using STBC, SFBC, and STFBC techniques under various fading channels. The performance is evaluated through a number simulations using MATLab. Bhagat and Malhotra [21] described three types of channel estimation techniques (estimators), namely, least square (LS), minimum mean square (MMSE), and linear minimum mean square (LMMSE). These estimators are implemented in MATLab, and the performance of the two types of estimators LS and MMSE are compared in terms of BER and MSE.

Sharma and Kaur [22] demonstrated BER analysis of MIMO-OFDM using MATLab, which is a technique of integrating OFDM to MIMO system to improve spectral efficiency in conjunction with ISI reduction, incorporating various modulation schemes. Furthermore, they compared the results with the performance of conventional OFDM (C-OFDM) system and reported an improvement in BER with MIMO-OFDM system. A wavelet-based OFDM model was developed by Kumar and Anuradha [23]. They analyzed and compared the performance of the model against the DFT-based OFDM in LTE system using MATLab. Based on the simulation outcomes, they propose the wavelet OFDM in place of the DFT OFDM in LTE system.

Divya [24] analyzed the BER performance of BPSK modulation and OFDM-BPSK system over Rayleigh fading channel, and also compared the performance of BER of BPSK over AWGN and Rayleigh channel. The simulation results show that the simulated BER is in good agreement with the theoretical BER for BPSK modulation. Sharma and Srivastava [9] developed a user interface using GUI tool of MATLab to facilitate analyzing the performance of OFDM system. In particular, they analyzed the BPSK, QPSK, and QAM techniques in OFDM system, where the Rayleigh fading channel and multipath fading channels are used as a communication channel. Ghorpade and Sankpal [10] designed and simulated an OFDM transmitter and receiver using MATLab.

Islam [16] presented a comparative performance analysis of OFDM system using comb-type pilot-based channel estimation algorithm over frequency selective multi-path fading channels. He used MMSE for the channel estimation at pilot and data frequencies. The OFDM simulation has been carried out with MATLab and the performance is analyzed in terms of BER for various signal mapping and channel conditions. Wang et. al. [25] developed a blind channel estimation method for trailing zero (TZ) OFDM systems in the frequency domain, which possess some favorable features compared to conventional cyclic prefixed (CP) OFDM systems. The MATLab simulation results demonstrate that the performance of the method is overwhelmed the performance of the existing channel estimation methods and its resistance to channel overestimation.

In conclusion, it is observable that simulation of OFDM system using MATLab is a powerful approach that can play a big role in identifying the most-efficient and cost-effective techniques for OFDM system (e.g., cannel estimation techniques, modulation techniques, equalization techniques, etc.). 


\section{OFDM SYSTEM MODEL}

The main concept and major advantage of OFDM systems is to transmit symbols over multiple orthogonal subcarriers, so inverse fast Fourier transform (IFFT) is performed with transmitted symbols at the transmitter, and fast Fourier transform (FFT) is performed with received symbols at the receiver. Another advantage is to convert a frequency-selective wideband channel into several frequency-flat narrow-band channels. Thus, the complexity of the receiver for OFDM systems is much simpler than that of single-carrier receivers. OFDM systems are sensitive to channel variation, which induces inter-carrier interference (ICI) by destroying the orthogonality between subcarriers [1-4].

\subsection{Basic Principles of OFDM}

Defining the symbol transmitted over the $k$ th subcarrier frequency as $s(k)$, and $x(n)$ as the received symbol at the $n^{\text {th }}$ time index, the output of IFFT at the transmitter is given by [26]:

$$
x(n)=\frac{1}{\sqrt{N_{s}}} \sum_{k=0}^{N_{s}} s(k) e^{j 2 \pi k n / N_{s}} \quad \text { for } n=0,1, \ldots, N_{s}-1
$$

where $n$ and $k$ represent the time index in an OFDM symbol period and subcarrier index, and $N_{s}$ represents the number of subcarriers. In Eqn. (1), the multi-path channel effects and noise are not included. Thus, the received OFDM symbol at the $n^{\text {th }}$ time $r(n)=x(n)$. On the receiver side, the output of FFT at the $k$ th subcarrier is given by [26]:

$$
y(k)=\frac{1}{\sqrt{N_{S}}} \sum_{n=0}^{N_{S}-1} r(n) e^{-j 2 \pi k n / N_{S}}
$$

Substituting Eqn. (1) into Eqn. (2), then after rearrangement Eqn. (2) becomes [26]:

$$
y(k)=\frac{1}{\sqrt{N_{S}}} \sum_{n=0}^{N_{S}-1} \sum_{q=0}^{N_{S-1}} s(q) e^{j 2 \pi n(q-k) / N_{S}}
$$

The above equation shows that the received symbol $y(k)=s(k)$ is not affected by the symbols from other subcarriers indicating the orthogonality between the subcarriers. The waveforms of the FFT of Eqn. (3) of different subcarriers are overlapped, but for one particular subcarrier, the sidelobes from other subcarriers are equal to 0 , which explains the orthogonality between the subcarriers.

\subsection{Fundamental Pilot Allocation for OFDM Systems}

Due to the orthogonality between the subcarriers in OFDM systems, different pilot allocation schemes can be adopted [1, 16]. In what follows, we explain some of the fundamental pilot allocation techniques; namely, block-type, comb-type, and lattice-type pilot allocation.

\subsubsection{Block-type pilot allocation scheme}

In block-type pilot allocation scheme, pilots are periodically inserted into all subcarriers in the frequency domain, so the channel frequency response for each subcarrier can be estimated. Fig. (1) shows the diagram of block-type pilot allocation. The quantity $t_{p}$ in Fig. (1) represents the time 
sampling period of pilot symbols, which must be much smaller than the inverse of Doppler frequency $\left(f_{d}\right)$ or coherence time. Henceforth, the block-type pilot allocation is designed for the frequency selective channel. In this scheme, more pilots may be employed if the channel variation between consecutive OFDM symbols increases.

\subsubsection{Comb-type pilot allocation scheme}

The idea behind the comb-type pilot allocation scheme is similar to the block-type scheme except that it combats the time variations of the channels between OFDM symbols. Fig. (2) illustrates the diagram of the comb-type pilot allocation scheme. The pilots are inserted in several particular subcarriers across all the time. In this scheme, the frequency sampling period of pilot symbols $\left(f_{p}\right)$ must be much smaller than the coherence bandwidth for the reliable channel estimates.

\subsubsection{Lattice-type pilot allocation scheme}

As compared to the above allocation schemes, the pilots are scattered over the time/frequency domains to keep track of the frequency selectivity and time variation of the channels. The parameters $t_{p}$ and $f_{p}$ must be much smaller than the coherence time and the coherence bandwidth. Fig. (3) illustrates the scheme of lattice-type pilot allocation. This scheme provides better tradeoff between the overhead of pilots and performance compared to the other conventional pilot allocation schemes. This is because it does not insert pilots across all the time as in comb-type pilot allocation scheme and does not insert pilots either in every subcarrier as in block-type pilot allocation scheme. Thus, this scheme can estimate the less selective channels in the time and the frequency with the reasonable number of pilots. 


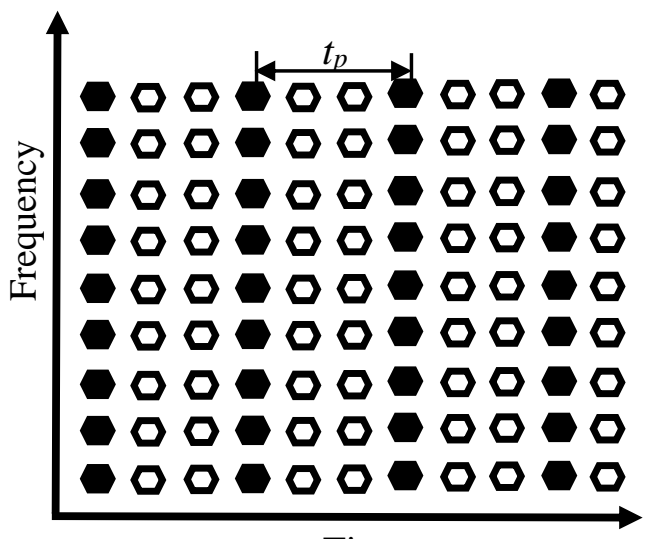

Time

Fig. (1). Block-type pilot allocation scheme.

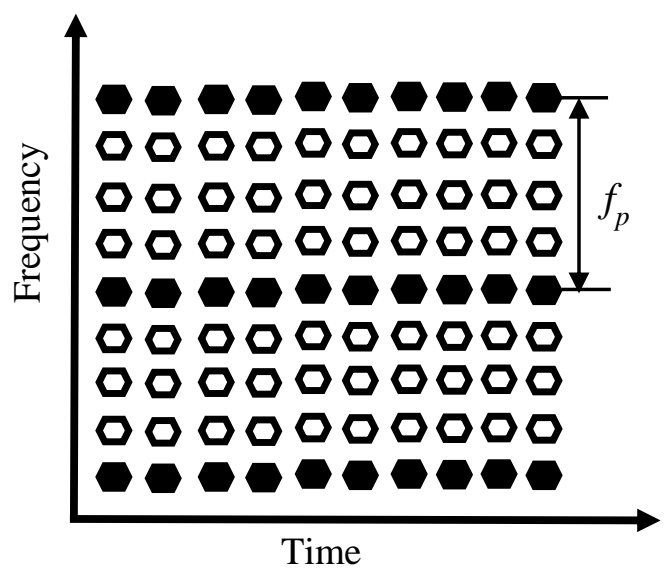

Fig. (2). Comb-type pilot allocation scheme.

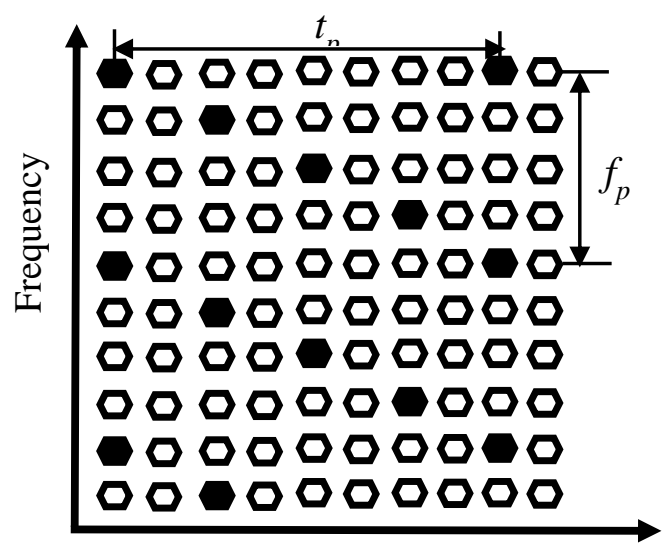

Time

Fig. (3). Lattice-type pilot allocation scheme.

\section{Channel Estimation Over Quasi-Static Channels}

In this paper, we concern with two different types of the channel estimation: the decision-directed and pilot-based, which are briefly introduced below [26]. The channel for each path is assumed to be quasi-static or Rayleigh channel, which means that the channel follows Rayleigh probability density function. Quasi-static or Rayleigh channel (also known as quasi-static Rayleigh channel (QSRC)) means that the channel is constant for a block of transmission and this constant within blocks vary independently. So, the constant for a block may be different from the constant in another block. This constant value is the combination of channel's constant gain and constant phase values $[15,16,24]$.

Before proceeding with channel estimation, it is important first to detect the signal. There are two main types of channel detection, these are: coherent detection and non-coherent detection. During the detection stage, an estimate of the channel phase and attenuation is recovered, so that it is possible to reproduce the transmitted signal and demodulate. Coherent detection can accomplish a better data rate and a better performance as compared to non-coherent detection techniques, at the price of acquiring accurate channel estimates. This indicates the need for accurate and effective channel estimation techniques [21]. 


\subsection{Decision-Directed Channel Estimation}

The decision directed (DD) channel estimation technique employs the training sequences for the initial channel estimation, and then employs the detected symbols using the previous channel estimate in the $(n-1)^{\text {th }}$ OFDM symbol period to predict the $n^{\text {th }}$ channel estimate. This method can achieve very high data rate at the expense of adding more processing delay [26].

Fig. (4) shows a block diagram of the DD channel estimation, and in order to correct the effect of fading by the receiver, equalization is performed at the receiver, which is the process that is used to measure the channel response and use this information to correct the received signal. Equalization is based on the channel properties and modulation schemes. The DD channel estimation can be easily integrated with LS or MMSE channel estimation as an equalization technique. The LS and MMSE techniques are explained below [27].

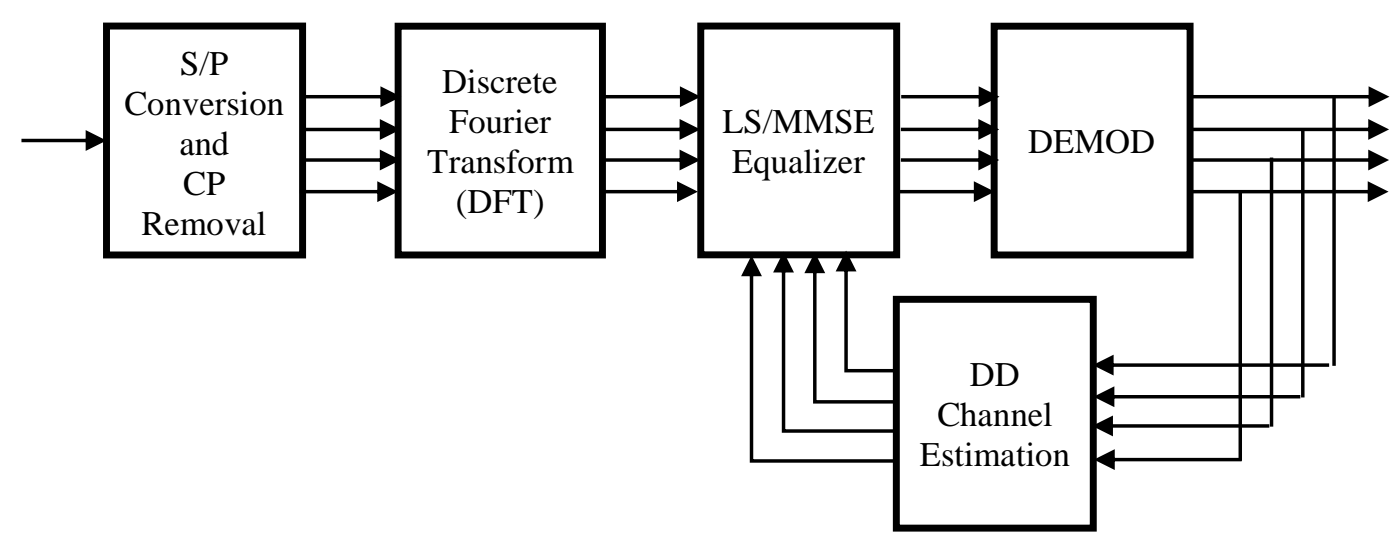

Fig. (4). Block diagram of DD channel estimation for OFDM systems.

The DD channel estimation suffers from two basic problems: The assumption of correct data detection and the use of outdated channel estimates. When the channel is varying very slowly; the use of outdated channel estimates does not create a serious problem. However, the outdated channel estimates for the previous OFDM symbol are no longer valid for the use of the data detection in the current OFDM symbol when the channel starts varying faster. For this reason, the error in data detection and channel estimation builds up to make the system performance sometimes unacceptable [21].

\subsection{Pilot-based Channel Estimation}

Pilot-based channel estimation is the most common technique for channel estimation, which is based on pilot symbol-assisted modulation (PSAM). It depends on the transmission of pilot symbols which are known at the receiver. The symbols are inserted into the data stream and then transmitted periodically over the radio channel in time and frequency domain, and are spread over the entire transmission bandwidth. In the receiver side these pilot symbols are analyzed to achieve channel estimation and estimate the data symbols at the receiver.

In the pilot allocation schemes discussed in the previous section, the pilots will be inserted in the time or frequency domain to estimate the channels for a particular time instant or subcarriers. The channels for data symbols are unknown to the receiver except the pilot channel estimates. The interpolation techniques are needed to estimate the channels between subcarriers or time slots. 
The interpolation techniques which are described below are performed based on the channel estimates obtained by LS or MMSE channel estimation [27, 28]. Fig. (5) shows a block diagram of pilot-based interpolation channel estimation for OFDM systems.

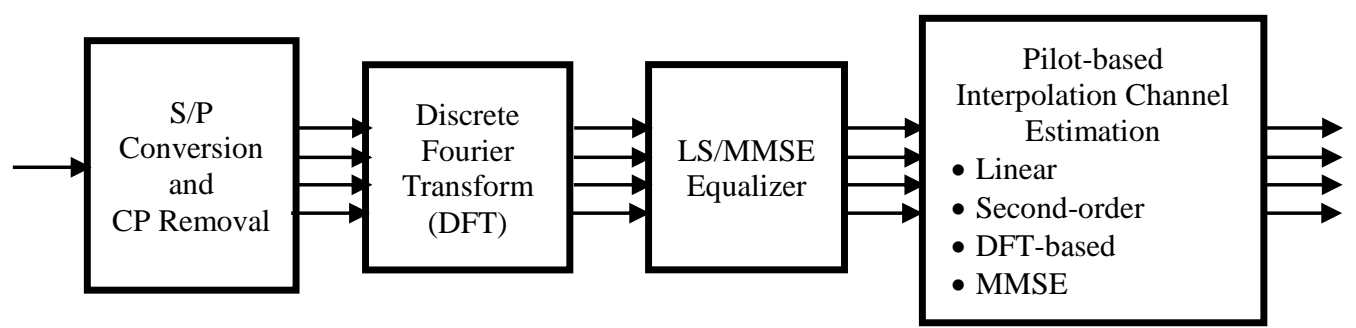

Fig. (5). Block diagram of pilot-based interpolation channel estimation for OFDM systems.

\subsubsection{Interpolation Channel Estimation Techniques for OFDM Systems}

In this paper, the performance of the following interpolation techniques are discussed and compared $[16,26]$.

Linear interpolation. Linear interpolation is a simple method to efficiently perform piecewise constant interpolation. Simply, it estimates the channels between two pilots with the aid of linear approximation. The optimal number of pilots for a given BER can be computed ahead for OFDM systems using the numerical evaluation.

Second-order interpolation. The idea behind second-order interpolation is similar to the linear interpolation except as its name indicates, it employs a second-order approximation. The MSE performance in the second-order interpolation is expected to be better than that of the linear interpolation with increasing complexity, i.e., more computing time.

DFT-based interpolation. The DFT-based interpolation technique is developed as a result of the Fourier transform of the channel impulse response. The DFT-based interpolation effectively eliminates the effects of noise outside the maximum channel delay spread or the length of multipath channel $L$. The implementation of the DFT-based interpolation is very straightforward compared to the linear or second-order interpolation. However, in this technique, the length of multipath channel must be known to the receiver. The DFT based interpolation technique requires FFT and IFFT computation as well as the length of multipath. Therefore, it appears as an excellent approach for channel estimation for OFDM systems.

MMSE interpolation. Compared to the above discussed interpolation techniques, MMSE interpolation may be considered as one of the most effective method for channel estimation between pilots with the aid of statistical information on the channels such as: the channel correlation and SNR, as well as additional computation of the matrix inversion.

\section{THE OFDM MODEL}

The main components of the OFDM model are shown in Fig. (6), which shows that the binary input data at the transmitter is first grouped and mapped using certain modulation technique (e.g., Quadrature Phase Shift Keying (QPSK), 16QAM, and 64QAM) in "Signal Modulation", and then the modulated data undergoes serial-to-parallel (S/P) conversion. 
After adding pre-known pilot symbols, so that a frequency domain transmitted data is formed. Afterwards, the IDFT block transforms the data sequence into time domain signal. Following the IDFT block, cyclic prefix (CP) to preserve the orthogonality of the subcarriers and to prevent ISI is then added to forming vector. After parallel to serial conversion $(\mathrm{P} / \mathrm{S})$, digital to analog conversion (D/A) and low pass filtering (LPF), the transmitted signal will pass through the frequency selective time varying fading channel with additive white Gaussian noise (AWGN) $[26,28]$.

In the receiver, reverse operations are performed which includes analog-to-digital (A/D) conversion, serial-to-parallel (S/P) conversion, CP removal, DFT computation, equalization and channel estimation using either DD or pilot-based channel estimation, parallel-to-serial conversion, and demodulation, respectively. Thus the model allows simulating the OFDM signal formulation, transition, and receiving.

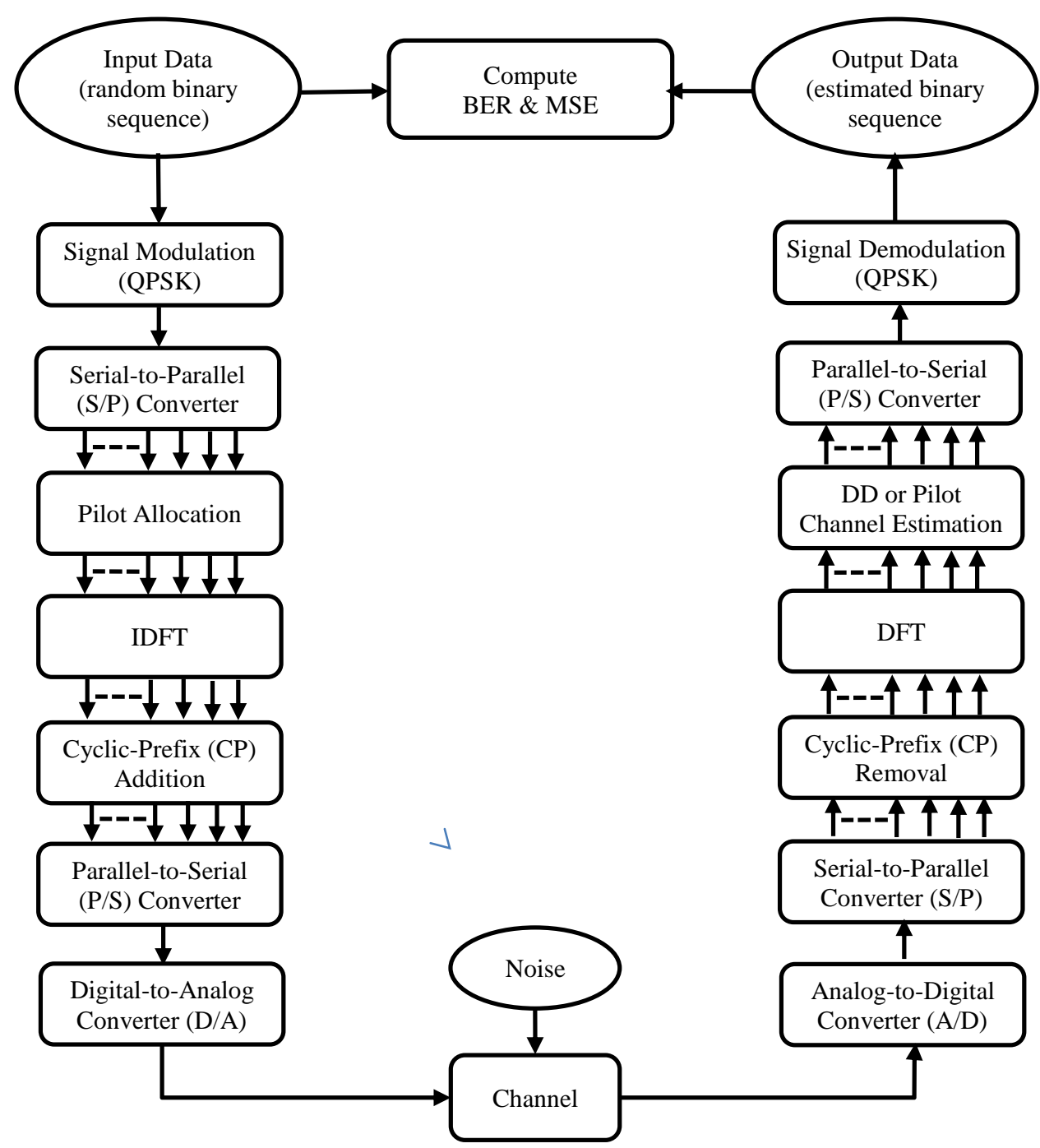

Fig. (6). Block diagram of the OFDM model. 


\section{RESULTS AND DISCUSSION}

In order to compare the performance of the channel estimation techniques discussed in this paper, namely, DD, linear interpolation, second-order interpolation, DFT-based interpolation, and MMSE interpolation, over quasi-static channels demonstrate, a number of simulations were performed using MATLab. In these simulations, we consider an un-coded OFDM system with the number of subcarrier $N_{s}=64$, and uniformly placed pilots $N_{p}=8$. The signal is QPSK modulated. The channel is generated using Jakes' model with a normalized Doppler frequency $f_{d} T_{\mathrm{OFDM}}=10^{-4}$, the length of which is $L=4$ with an exponential power delay profile. Furthermore, the channel taps for different delays are independent from each other.

Other input simulation parameters are: the MMSE detector is implemented at the receiver side, and the channel frequency responses of pilot subcarriers are estimated by LS estimation. The DD channel estimation send pilots across subcarriers in every 8 OFDM symbols. The BER and MSE for different techniques over an SNR range from 10 to $30 \mathrm{~dB}$ are shown in Fig. (7) and Fig. (8), respectively.

The simulation results in Figs. (7) and (8) show that the DD channel estimation has an excellent performance compared to other interpolation techniques. However, it added extra processing delay in the OFDM system, and is comparatively sensitive to channel variations between OFDM symbols. The simulation results also show that the MMSE interpolation outperforms other techniques in the BER and MSE performance except DD channel estimation.

However, DD also requires statistical information on communication channels such as frequency correlation and SNR, and matrix inversions must be performed for each OFDM symbol. It can be easily recognized that the performance of DFT channel estimation is poorer than that of MMSE with $4 \mathrm{~dB}$ loss in the MSE performance, but these two channel estimators (DD and DFT) demonstrate almost the same BER performance.

Moreover, the DFT channel estimation only requires the implementation of FFT and IFFT and also information on the length of multipath channel. Other techniques including linear and second-order interpolation experience error floors in BER and MSE. The error floors of BER are caused by the unreliable channel estimates over non-pilot subcarriers acquired by these interpolation techniques.

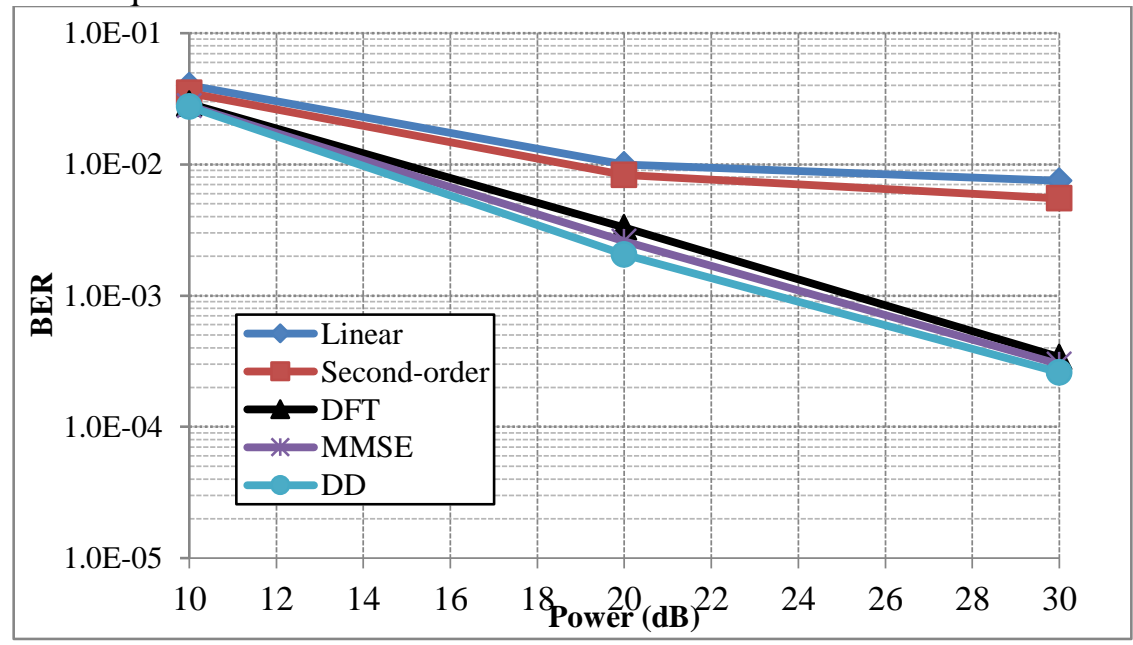

Fig. (7). BER performance of frequency domain interpolation techniques. 


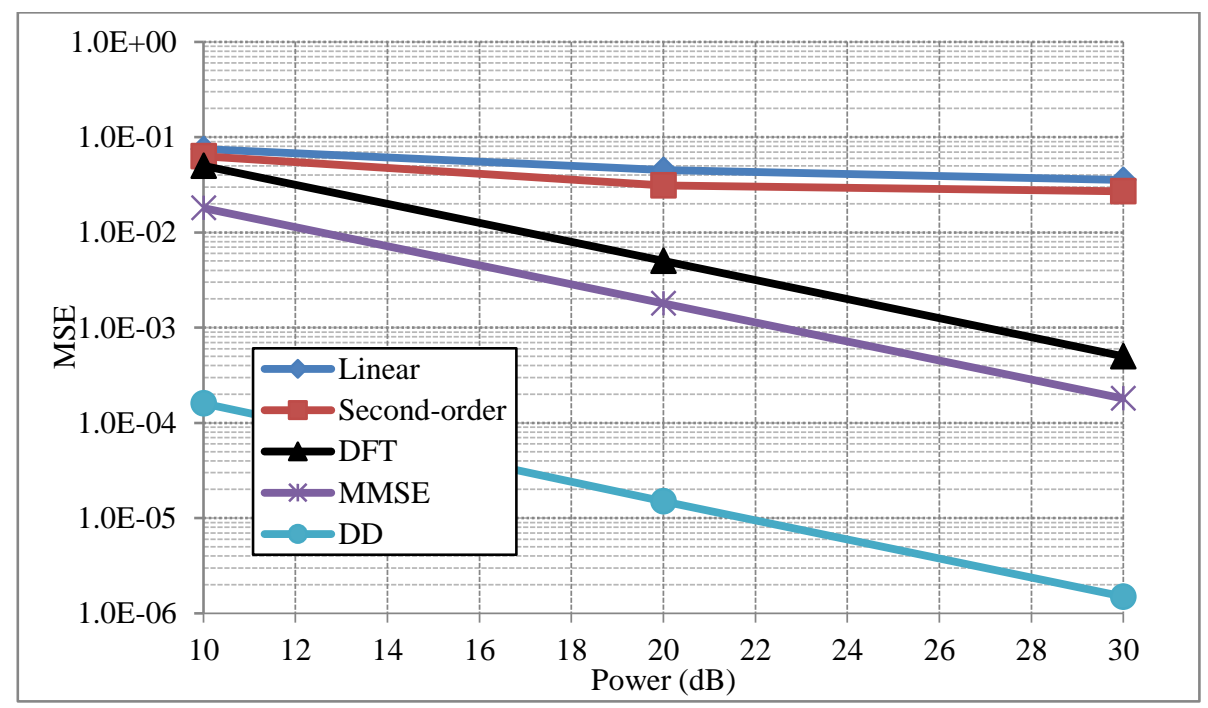

Fig. (8). MSE performance of frequency domain interpolation techniques.

\section{Conclusions}

The main conclusions of this paper are: (1) DD channel estimation has an excellent performance compared to other interpolation techniques in terms of BER and MSE over variable SNR. This is at the cost of extra processing delay in the OFDM system, (2) MMSE interpolation outperforms other interpolation techniques (linear, second-order, and DFT-based) in terms of both BER and MSE, and (3) DFT-based interpolation demonstrates an attractive tradeoff between complexity (time) and performance.

The main recommendations for future work are to enhance the user-interactivity of the MATLab simulator to enable more students, researchers, academicians, and professionals to use it easily. Implement and evaluate the performance of other pilot-based channel estimation techniques, pilot allocation techniques, and interpolation techniques under different wireless communication environments and system conditions.

\section{REFERENCES}

[1] Henrik Schulze and Christian Luders. Theory and Applications of OFDM and CDMA: Wideband Wireless Communications. John Wiley \& Sons, 2006.

[2] Yong Soo Cho, Jaekwon Kim, Won Young Yang, Chung G. Kang. MIMO-OFDM Wireless Communications with MATLAB, John Wiley \& Sons, August 2010.

[3] Mathuranathan Viswanathan. Digital Modulations using MATLab: Build Simulation Models from Scratch. E-book, June, 2017.

[4] Srishtansh Pathak and Himanshu Sharma. Channel Estimation in OFDM Systems. International Journal of Advanced Research in Computer Science and Software Engineering (IJARCSSE), Vol.3, No.3, pp. 312-327, 2013. 
[5] Elizabeth A. Thompson, Charles McIntosh, James Isaacs, Eric Harmison, Ross Sneary. Robot Communication Link Using $802.11 \mathrm{n}$ or $900 \mathrm{MHz}$ OFDM. Journal of Network and Computer Applications (JNCA), Vol. 52, Issue 6, pp. 37-51, June 2015.

[6] Jeffrey G. Andrews, Arunabha Ghosh, and Rias Muhamed. Fundamentals of WiMAX-Understanding Broadband Wireless Networking. Prentice Hall, Second Edition, 2007.

[7] Christopher Cox. An Introduction to LTE: LTE, LTE-Advanced, SAE and 4G Mobile Communications. John-Wiley \& Sons, March 2012.

[8] Mehdi Alasti, Behnam Neekzad, Jie Hui, and Rath Vannithamby. Quality of Service in WiMAX and LTE Networks. IEEE Communications Magazine, Vol. 48, Issue 5, May 2010.

[9] Deepak Sharma and Praveen Srivastava. OFDM Simulator Using MATLAB. International Journal of Emerging Technology and Advanced Engineering, Vol. 3, Issue 9, pp. 493-496, September 2013.

[10] S. S. Ghorpade and S. V. Sankpal. Behavior of OFDM System Using MATLAB Simulation. International Journal of Innovative Technology and Research (IJITR), Vol., No. 1, Issue No. 3, pp. 249 - 252, April - May 2013.

[11] S. Sadinov, P. Daneva, and P. Kogias. Description and Simulation of OFDM Reception Process Journal of Engineering Science and Technology Review, Vol. 7, No. 4, pp. 18-22, 2014.

[12] Orlandos Grigoriadis and H. Srikanth Kamath. BER Calculation Using MATLAB Simulation for OGDM Transmission. Proceedings of the International Multi-Conference of Engineers and Computer Scientists (IMECS), Vol II, Hong Kong, 19-21 March 2008.

[13] Kala Praveen Bagadi and Susmita Das. MIMO-OFDM Channel Estimation Using Pilot Carries. International Journal of Computer Applications (0975 - 888 (IJCA), Vol. 2, No. 3, May 2010.

[14] H. Sinha, R. Meshram, and G.R. Sinha. BER Performance Analysis of MIMO-OFDM over Wireless Channel. International Journal of Pure and Applied Mathematics (IJPAM), Vol. 118, No. 5, pp. 195206, 2018.

[15] Pratima Manhas and M.K Soni. OFDM Performance Evaluation under Different Fading Channels using Matlab Simulink. Indonesian Journal of Electrical Engineering and Computer Science, Vol. 5, No. 2, pp. 260-266, 2017.

[16] A. Z. M. Touhidul Islam. A Comparative Performance Study of OFDM System with the Implementation of Comb Pilot-Based MMSE Channel Estimation. International Journal on Computational Sciences \& Applications (IJCSA), Vol.3, No.6, pp. 45-53, December 2013.

[17] D. Khosla, S. Singh, R. Singh, and S. Goyal. OFDM Modulation Technique \& its Applications: A Review. Proceedings of the International Conference on Innovations in Computing (ICIC 2017), pp. 101-105, 2017.

[18] Fateme Salehi, Mohammad-Hassan Majidi, and Naaser Neda. Channel Estimation Based on Learning Automata for OFDM Systems. International Journal of Communication Systems, Vol. 321, Issue 12, August, 2018.

[19] Navjot Kaur and Neetu Gupta. Simulation and Analysis of OFDM and SC-FDMA with STBC using Different Modulation Techniques. International Journal of Advanced Research in Computer Engineering \& Technology (IJARCET), Vol. 4, Issue 11, pp. 4184-4189, November 2015. 
[20] Himanshi Jain and Vikas Nandal. A Comparison of Various Channel Estimation Techniques to Improve Fading Effects in MIMO over Different Fading Channels. International Journal of Current Engineering and Technology (IJCET), Vol. 6, No. 4, pp. 1382-1386, 2016.

[21] Kussum Bhagat and Jyoteesh Malhotra. Performance Evaluation of Channel Estimation Techniques in OFDM-based Mobile Wireless System. International Journal of Future Generation Communication and Networking (IJFGCN), Vol. 8, No. 3, pp. 53-60, 2015.

[22] Vishal Sharma and Harleen Kaur. On BER Evaluation of MIMO-OFDM Incorporated Wireless System. International Journal for Light and Electron Optics, Vol. 127, Issue 1, pp. 203-205, January 2016.

[23] N. Kumar and Anuradha. BER Analysis of Conventional and Wavelet Based OFDM in LTE using Different Modulation Techniques. IEEE Engineering and Computational Sciences, March 2014.

[24] M Divya. Bit Error Rate Performance of BPSK Modulation and OFDM-BPSK with Rayleigh Multiple Channel. International Journal of Engineering and Advanced Technology (IJEAT), Vol. 2, Issue 4, April 2013.

[25] Song Wang, Jinli Cao, Jiankun Hu. A Frequency Domain Subspace Blind Channel Estimation Method for Trailing Zero OFDM Systems. Journal of Network and Computer Applications (JNCA), Vol. 34, Issue 1, pp. 116-120, January 2011.

[26] Li Li. Advanced Channel Estimation and Detection Techniques for MIMO and OFDM Systems. PhD Thesis, University of York, UK, 2013.

[27] S. Patil and A. N. Jadhav. Channel Estimation Using LS and MMSE Estimators. KIET International Journal of Communications \& Electronics, Vol. 2, No.1, pp. 51-55, April 2014.

[28] Anwar Yousef Al-Tarawneh. An Improved Performance OFDM Channel Estimation Using PilotSymbol-Aided Technique. MSc Thesis, Mutah University, Jordan, 2015.

\section{Author}

Raghad K. Mohammed is serving as a member of academic staff at the Department of Basic Sciences, College of Dentistry, University of Baghdad (Baghdad, Iraq). She received her B.Sc degree in Computer Science from the Department of Computer science, Al-Rafidain University College (Baghdad, Iraq) in 2003, and her M.Sc degree in Computer Networks, Informatics Institute for Higher Studies, University of Technology (Baghdad, Iraq) in 2005. Her research interests include cryptography and steganography, image processing, and information and network security.

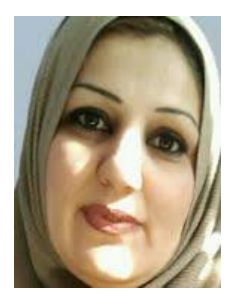

